\title{
L'histoire de l'orange fraîche ou celle des réseaux « ethniques » dans la région Cuenca de La Plata
}

\section{The story of fresh orange or the one of the ethnic network in the region of Cuenca de La Plata}

\author{
Delphine Mercier*, Marcos Supervielle* \\ Laboratoire d'Economie et de Sociologie du Travail - LEST (UMR 6123), \\ 35 Avenue Jules Ferry, 13626 Aix-en-Provence \\ Departamento de Sociología. FCS. Universidad de la República, Uruguay
}

\section{Résumé}

L'histoire de l'orange en Uruguay et Argentine est fortement liée à l'histoire de la migration de cet espace du monde. Chaque vague de migration a apporté un savoir-faire, une technique contribuant petit à petit à la création d'un produit : l'orange fraîche de contre-saison... Nous suivrons le fil des migrations pour présenter le développement de l'orange fraîche, de l'arrivée de Christophe Colomb à celle des «pieds-noirs » en 1962, les différentes populations ayant planté, développé, inventé le concept de «contre-saison » pour construire leur légitimité sur la scène agricole.

(c) 2007 Lavoisier, Paris. Tous droits réservés.

\section{Summary}

The story of orange fresh fruit from over-station in Uruguay and Argentina is strongly linked to the story of migration of this region of the world. Each wave of migration bringed a know-how, a technicity contributed at the creation of one product : the fresh orange over-station. We will follow the nit of migration to present the development of the fresh orange, from the arriving of Christopher Colombo at the one of "pieds noirs" in 1962, the different populations planted, deve-

\footnotetext{
*Adresses email : delphine.mercier@univmed.fr - marcos@fcssoc.edu.uy doi:10.3166/ges.9.271-281 (C) 2007 Lavoisier, Paris. Tous droits réservés.
} 
loped, invented the concept of "contre-saison" to built their legitimacy in the agriculture place.

(C) 2007 Lavoisier, Paris. Tous droits réservés.

Mots clés : Migrations, histoire, organisation agrumicole.

Keywords: Migrations, Chronology, citrus organisation.

Pedro Solari est un immigré italien, il a inventé l'orange fraîche de contre saison pour le marché européen. L'agrumiculture en Amérique du Sud s'est développée dans ce contexte, il s'agissait de produire pour le marché national, mais surtout d'exporter pour faire valoir un savoir-faire et sa connaissance de l'Europe.

En Amérique du Sud, l'histoire de l'orange est celle de la colonisation. Si les premières oranges ont été apportées par Christophe Colomb, c'est seulement au moment de leur indépendance que les pays d'Amérique Latine ont vraiment pu développer leur production. Pour des raisons de suprématie, les espagnols ont longtemps sauvegardé leur prééminence sur la circulation de ce fruit qui, pourtant, selon les textes retrouvés, était désigné pour avoir un bel avenir. Ainsi lors des premières plantations en 1520 des chroniqueurs du Santo Domingo déclaraient-ils : « ...les oranges se sont multipliées de façon si abondante que maintenant il y en a trop, le fruit est très bon que ce soit le fruit sucré comme l'amer ». .

C'est seulement au XVIII ${ }^{\mathrm{e}}$ siècle que la production d'oranges s'est développée. Cet essor est fortement lié à l'apogée du transport fluvial reliant les villes de la région de la Cuenca del Plata (région jouxtant le fleuve Rio Uruguay), située entre l'Argentine et l'Uruguay. Ensuite c'est au XIX ${ }^{\mathrm{e}}$ siècle que le secteur de l'agrumiculture se constitue complètement dans les deux pays, devenant un secteur reconnu. Il est évident que ce secteur et son développement ont été soumis aux modifications et grandes évolutions liées aux différentes étapes de développement de ces deux pays. Nous ne nous y attarderons pas pour nous attacher à l'histoire de l'orange vue à travers celles d'hommes et de femmes, d'acteurs qui selon les périodes ont voyagé, migré, se sont exilés et ont emporté avec eux et importé leurs savoirs-faire. Ces savoirs-faire, ils les ont développés sans cesse, les ont traduits, adaptés et adoptés dans certains cas pour faire face à de nouvelles situations. L'histoire de l'orange en Amérique Latine est donc avant tout liée à l'histoire de la colonisation et aux vagues de migration qui ont peuplé cette région du monde.

Dans cet article nous présenterons les conditions de l'évolution du système complexe des agrumes, en démontrant les arrangements sociaux qui ont été construits et ont contribué à consolider la chaîne de production-consommation complexe que nous connaissons aujourd'hui. Nous le ferons en suivant un fil chronologique, celui de l'histoire des différentes migrations puisque c'est celui qui nous est apparu comme le plus significatif.

Voici les trois « temps » que nous avons repérés, parallèles à trois vagues de migration : l'arrivée des premières oranges dans le bassin Cuenca del Plata, la période de l'hégémonie de Salto, et enfin l'émergence d'un système complexe agrumicole dans la région de Paysandú. On notera au cours de cette chronologie que les acteurs de l'orange dans cette région du monde ont toujours été des acteurs extérieurs au pays : tantôt portugais, basques, italiens et «pieds noirs ».... L'activité agrumicole ne s'est pas développée dans un espace vierge en termes d'agriculture. Cet espace était traditionnellement marqué par l'élevage qui prédominait et qui était clairement le pré-carré des populations plus anciennement arrivées. 
Comme nous le verrons, ce découpage de la naissance à la stabilisation de l'activité agrumicole est complètement articulé aux acteurs et aux réseaux qu'ils ont su constituer.

Les différentes vagues de migration, créant des arrivées massives ou intensives de population d'Europe, ont occupé des activités différentes (arboriculture, commerce...). Chaque dernier arrivé devant occuper des scènes productives laissées vacantes par les précédents.

\section{L'arrivée de l'orange dans le Bassin Cuenca del Plata. La ville de Salto et sa place économique fragile pendant la période coloniale et pendant le XVIII ${ }^{\mathrm{e}}$ siècle.}

On considère que les agrumes sont originaires d'une vaste région comprise entre l'Inde, la Chine et l'archipel Malayo. En suivant les routes commerciales, la production d'agrumes s'est étendue sur les territoires de Perse, de Mésopotamie, et ensuite de Palestine pour finalement aboutir au nord de l'Afrique et dans l'espace méditerranéen. Les premiers agrumes étaient des «cidres» et des «oranges amères». C'est au cours de l'ère chrétienne qu'apparaissent les oranges douces qui ont été appelées dans le bassin de la Cuenca del Plata les oranges chinoises (XIX ${ }^{\mathrm{e}}$ siècle). De façon concomitante apparurent les mandarines. Aujourd'hui encore se discute le fait de savoir si les oranges précolombiennes autochtones ont existé avant ou après l'arrivée des conquistadors. Mais en ce qui concerne les oranges actuelles elles furent apportées par les conquistadors.

Christophe Colomb, lors de son second voyage en Amérique, apporte des oranges qui seront plantées sur l'île Hispagnola (aujourd'hui Haïti). On trouve, d'ailleurs, dans les récits situés entre 1514 et 1525 des citations telles que “.... le naturaliste Oviedo y Valdez relate dans sa chronique de Santo Domingo entre 1514 et 1525 , ...les oranges se sont multipliées de façon si abondante, que maintenant les oranges surabondent, le fruit est délicieux que ce soit l'orange sucrée comme l'orange amère... ${ }^{1}$. Selon le récit de Bernal Díaz de Castillo dans le livre «Verídica Historia de la Conquista de Nueva España», Hernán Cortés les apporte sur le continent.

Cependant, le contrôle douanier et la fermeture économique opérés par la Couronne Espagnole influèrent de façon négative sur l'expansion des agrumes en Amérique Latine, ceci pendant toute la domination espagnole. En effet, pendant pratiquement deux siècles et demi, le commerce de l'empire se réalisait au travers des Caraïbes, Séville et Cadiz. C'est une des raisons pour lesquelles le développement de la colonisation tarda tant dans les régions du sud, particulièrement dans la région qui nous intéresse : la Banda oriental del Uruguay. Elle fut la dernière région colonisée par la Couronne d'Espagne des Amériques. Malgré ce retard, la proximité avec les missions jésuites qui s'étaient installées dans ce qui est aujourd'hui le nord de l'Argentine et le Paraguay ont rapproché la plantation des oranges de cette région. Les jésuites développèrent la culture de l'orange entre autres activités agricoles. Patricio Fernandez soutient que les jésuites encouragèrent "l'arboriculture, en fournissant des semences et des prêts en argent pour les voisins qui souhaitaient développer leur production, les orangers paraguayens des Missions sont connus dans la région... ». ${ }^{2}$

Les jésuites dominaient déjà l'art de la plantation des agrumes en vue d'une production commerciale. On peut lire dans « l'observation sur l'agriculture » que le prêtre Jose

\footnotetext{
${ }^{1}$ Chronique de Santo Domingo entre 1514 et 1525.

${ }^{2}$ Op.cité
} 
Manuel Perez Castellano réalise en 1813 que cette expertise est effectivement bien avancée. Il y explique les différents problèmes liés à cette production et culture arboricole.

Il décrit d'abord les problèmes liés aux pépinières et relate ensuite l'importance de choisir les meilleures terres pour ce type de plantation. Il s'attarde également sur les différentes façons de planter et de transplanter les arbres. A titre d'exemple, il note l'exigence de distancier les arbres en précisant un espacement minimal. Il évoque également la nature des engrais nécessaires, les besoins en termes d'arrosage et les techniques conjointes pour réaliser au mieux l'irrigation. Dans son écrit, il mentionne des questions relatives à l'ensoleillement des arbres, et surtout à la question de la protection des vents. Démontrant une réelle maîtrise technique, il fait référence à l'utilisation de «greffes » pour produire des oranges sucrées sur des pieds d'orangers amers ${ }^{3}$. Enfin, des éléments sur l'âge des arbres, les mois de production et les maladies sont également notés.

Mais les oranges arrivèrent également dans l'espace de la Banda Oriental del Rió Uruguay par le biais des portugais. Ces derniers, cherchant à agrandir continuellement leur territoire au-delà de ce qui leur avait été accordé dans le cadre du Tratado de Tordecillas marquant la division des terres d'Amérique avec l'Espagne, fondent la ville de Colonia en 1680 en Uruguay. Cette ville passa plusieurs fois des mains des portugais à celles des espagnols, mais les portugais qui avaient construit la ville plantèrent des orangers sur la place principale. Aujourd'hui encore, on peut trouver leur descendance.

C'est donc progressivement, au cours de ces deux siècles, que l'orange s'installe en Uruguay et en Argentine, mais elle reste un fruit exotique jusqu'au début du XIX ${ }^{\mathrm{e}}$ siècle. Comme nous allons le voir dans la partie suivante, c'est seulement à partir de 1850 que débuteront la production d'oranges et sa commercialisation intensive.

\section{La naissance et la consolidation de l'Agrumiculture commerciale. La période de l'hégémonie de Salto.}

Ce démarrage de l'orange se réalisera à Salto. Avant de décrire les éléments qui ont contribué au développement de l'agrumiculture dans cette région il est utile de préciser dans quel contexte de développement se situe la région.

C'est au cours du XIX ${ }^{\mathrm{e}}$ siècle que cette ville prendra toute son importance. Elle naît autour de l'activité fluviale qui se développe dans la région. Véritable carrefour entre l'Uruguay, l'Argentine, le sud du Brésil et le Paraguay, l'activité fluviale commerciale connaît tout son essor et Salto est la ville phare de cette période. Le transport est sûr, rapide, permettant la circulation de grosses quantités ${ }^{4}$. Pendant cette période, on observe une grande fluidité de circulation entre les pays, il n'y a pas de lois de cabotage, ni de restrictions.

L'arrivée du chemin de fer en 1868 et en 1874 à Salto (faisant de Salto le point de départ et d'arrivée du Nord-Est) va renforcer la position stratégique de cette ville, mais va par contre fragiliser le transport fluvial. C'est à partir de 1920, avec la consolidation du réseau routier (construction de routes, de ponts...) qui renforce l'intégration des différentes villes au niveau national, que Salto perd de son prestige. Le réseau routier va mettre fin pratiquement au transport fluvial et au chemin de fer, et va changer les espaces de circulation. La consolidation du

\footnotetext{
${ }^{3}$ La plantation de mandarines a débuté à Salto en 1864 à partir de graines venues de Rio de Janeiro.

${ }^{4}$ En 1852 : création de la première Compagnie Nationale de Navigation à Salto.
} 
réseau routier des deux côtés (Argentine et Uruguay) fera perdre à la région du Rio Uruguay sa force, sa place dans l'espace économique régional le réduisant à un espace frontalier, de fermeture économique et sociale de chaque pays sur lui-même. Cette fermeture ouvre la porte à une future politique de substitution d'importation des deux côtés pour les deux pays.

L'agrumiculture à Salto apparaît donc à la période hégémonique du Port de Salto, en 1875, fournissant à la fois le marché de Montevideo et le marché de Buenos Aires. La production d'agrumes va connaître trois périodes assez classiques de développement : la période de production réduite à la consommation et à la décoration ornementale, la période de l'activité commerciale « secondaire » en se développant dans des espaces de plus en plus éloignés de la consommation, et enfin, la période de production extensive avec une orientation vers des grands marchés exigeants, à destination de consommateurs dont les goûts sont diversifiés. Le passage d'une étape à l'autre est complexe pour les producteurs car il implique à chaque fois des avancées technologiques en termes de production, de prise en compte de l'évolution des goûts, et de différenciation des produits pour satisfaire les différents consommateurs. Ces avancées technologiques impliquent surtout de profondes transformations dans l'organisation du travail, soit la transition d'une production artisanale vers une production industrielle.

Le passage de la production domestique à une production commercialisée implique et exige surtout la plantation d'arbres à différentes étapes, afin d'obtenir une surproduction qui soit commercialisable. Il semble nécessaire alors d'avoir une véritable expertise dans les conditions de plantation des arbres.

\subsection{La production d'oranges : le pré carré des immigrants}

La production d'oranges n'a pas été impulsée par l'oligarchie familiale de propriétaires terriens éleveurs de Salto ${ }^{5}$, mais plutôt par une nouvelle classe d'immigrants à partir de 1875. Cette portion de la population nouvellement arrivée d'Europe, souhaitant « faire sa place » et à la recherche d'une ascension sociale s'est orientée plutôt vers des activités qui permettaient de conjuguer créativité, innovation et formation appliquées à la production. Pour cela un secteur agricole, celui de l'arboriculture, demeurait non exploité. A Salto et au Nord de Salto, ce sont les immigrants basques qui plantèrent les premiers arbres fruitiers. Le point de départ est celui de la plantation des ceps de vigne et la production du vin. Avec la plantation fruiticole, mais également avec une production végétale, c'est un nouveau tournant qui est pris dans l'agriculture de cette région.

Nous pouvons illustrer cette évolution avec l'histoire de Juan Gautron, basque d'origine, qui fut embauché pour le travail dans les vignes et dans les caves. Toute sa famille viendra avec lui de Cognac. Son fils Esteban vivra en Uruguay et repartira en France pour réaliser des études d'agronomie. En 1898, de retour en Uruguay, face à l'avenir prometteur du marché des agrumes qui s'ouvrait à Buenos Aires, il réussit à mettre en œuvre une innovation technologique en réalisant plusieurs greffes et en créant le premier vivier d'arbres fruitiers important du pays.

L'autre fondateur de la production agrumicole commerciale à Salto et en Uruguay fut Pietro Solari qui arrive d'Italie jusque dans la région de La Plata en 1850. Rapidement

\footnotetext{
${ }^{5}$ Salto était alors protégé des guerres civiles qui se déroulaient entre Buenos Aires et Montevideo.
} 
il décida de s'installer dans la région du Rio Uruguay. En 1852, il s'installe enfin à Salto et y ouvre une boutique, dans le but de commercialiser la production de fruits et de viandes. C'est la naissance de cette activité commerciale qui se développa avec les différentes générations de Solari (de père en fils) qui fait de Pedro Solari, le neveu du premier immigrant, une référence encore aujourd'hui en termes de production agrumicole dans la région. A partir de cette activité commerciale, les Solari ont diversifié leur activité vers la production de volaille, mais surtout vers l'agrumiculture.

C'est la combinaison de compétences techniques et marchandes, basée sur un relationnel construit grâce à un réseau d'acteurs présents dans les deux villes de Buenos Aires et de Montevideo qui constituera le moteur du développement de ce secteur. N'oublions pas d'autres facteurs primordiaux comme les conditions climatiques adaptées à la production des agrumes et la place stratégique de la ville de Salto à l'époque qui créèrent des conditions idéales pour l'installation et la consolidation d'un nouveau secteur productif'.

Pourtant, dans ce contexte privilégié, il y avait deux handicaps notoires :

- L'intégration des « agrumes » dans le panier de consommation des familles argentines et uruguayennes.

- Pour l'Uruguay, la concurrence avec les zones de production d'oranges argentines.

Cette période hégémonique dura jusqu'en 1925, période à laquelle commencent les premières alertes de surproduction sur le marché uruguayen. C'est donc à ce moment là que se fait sentir la nécessité absolue de trouver de nouveaux marchés, en particulier européens. Dans ce contexte de grande concurrence, se pose pour les producteurs de Salto un nouveau défi, celui de la recherche de la qualité ${ }^{7}$. Cet investissement dans la qualité a été anticipé par Pietro Solari qui la considérait comme la condition pour faire face à la concurrence. Ce cheminement est marqué par un double mouvement : d'une part, celui d'une institutionnalisation de l'activité agrumicole, qui à l'époque n'est pas encore consolidée ; d'autre part, la volonté d'améliorer la production. L'institutionnalisation de l'activité est impulsée par le bais d'activités de promotion, puis par la mise en place de mécanismes corporatifs de défense de la production d'agrumes. En 1918, est créée la Liga de Fomento Rural, puis la Liga de Fomento de Salto, suivies en 1936 par la Cooperativa Citricola Saltena et l'Union Naranjera Saltena. Cette institutionnalisation permet notamment de faire valoir des positions auprès de l'Etat, de créer un rapport de force favorable face aux éleveurs très influents dans le pays.

De façon conjointe à cette recherche de qualité, les producteurs souhaitent également améliorer la production. En 1932, Pietro Solari réalise un long séjour aux Etats-Unis, en Californie et en Floride, avec cet objectif. Au cours de son séjour, il passera du temps aussi bien dans des centres de recherche universitaires en agronomie, que dans les pépinières. De ce voyage, Solari va rapporter dans ses valises, et surtout dans ses pratiques, des innovations techniques

\footnotetext{
${ }^{6}$ En 1919, la récolte d'oranges de Salto atteint les 33 millions de fruits, parmi lesquels 6 millions furent embarqués pour Buenos Aires. La majorité provient de l'établissement de Solari, Cuatro Bocas, dans lequel il a une plantation de 44000 arbres.

${ }^{7}$ Dans le livre intitulé "Centenario" (Les livres en hommage au centese le denomina así al libros que se elaboró en homenaje al centenario de la Independencia en 1930) on signale « Les oranges de Salto sont connues dans tout le pays pour leurs goûts succulents, pouvant concurrencer as naranjas de Salto gozan de merecida fama en todo el país por su sabor exquisito, pudiendo competir ventajosamente con la paraguaya, correntina y brasilera.
} 
notoires qui vont bouleverser la production et surtout intégrer des processus techniques permettant la valorisation de l'agrumiculture. On peut d'ailleurs qualifier cette phase comme celle de l'intégration de techniques, d'outillages, de produits permettant l'augmentation de la valeur ajoutée dans la chaîne de production de l'orange. On peut en citer quelques unes parmi celles qui contribueront à modifier le processus de production en amont, les qualités du produit, soit sa nature, sa résistance et son mode de finition. Ainsi se développent :

- Les techniques de déverdissage des oranges.

- Les techniques pour combattre le chancre ${ }^{8}$ des agrumes.

- L'introduction de cinquante variétés nouvelles d'oranges et de mandarines (parmi ces dernières des oranges sans pépins, les Satsumas, qui seront par la suite très appréciées au niveau national et international).

- La mécanisation des différents rinçages.

- La mécanisation de la sélection des fruits.

- La mécanisation de l'emballage des fruits.

Solari, grâce à ses connaissances en ingénierie et aux observations réalisées aux EtatsUnis, conçoit la première station d'emballage (packing) en Uruguay, il a également conçu dans ses ateliers une des premières machines d'extraction de jus.

C'est à cette période que la production de l'orange va prendre un nouveau tournant pour devenir une activité de production plus complexe alliant à la fois croissance et technicisation de l'activité.

\subsection{L'apparition de nouveaux acteurs}

Un véritable marché s'est progressivement constitué exigeant la présence d'intermédiaires, que ce soit dans le commerce des emballages, dans le transport ou dans la manipulation de la marchandise. Des stratégies différentes vont donc être mises en œuvre pour mener à bien cette évolution. Cette division du travail, et la multiplication des échelons intermédiaires, va s'opérer de façon distincte et va permettre la mise en œuvre d'une chaîne de production globale, de la culture à la commercialisation.

Solari, avait, dès le départ, intégré une maison commerciale. Dans ce cas, une partie de cette division du travail se réalisa donc à l'intérieur même de la firme. Des entreprises se développèrent par ailleurs pour réaliser et organiser à Montevideo le transport, l'arrivage, la commercialisation sur le marché et le retour des emballages, essentiellement des caisses.

Dans le cas d'autres familles de Salto, ce sont les frères d'une même famille qui s'installèrent à la fois à Salto et à Montevideo afin d'organiser la chaîne de façon efficace, mais surtout afin de diminuer le risque permanent provenant des impondérables de la production, du transport et des fluctuations du prix d'un produit dont la conservation était courte.

Dans le cadre de ce processus, de nombreux commerçants sont devenus des producteurs, tout comme des producteurs ont intégré l'aspect commercial. Ils le faisaient tous afin de réduire les échelons intermédiaires et pour produire en accord avec leurs impératifs commerciaux.

C'est dans les années 1930 que plusieurs entreprises commerciales tentent l'exporta-

\footnotetext{
${ }^{8}$ Le chancre des agrumes est une bactérie qui abîme la peau des fruits. Si la qualité gustative et sanitaire des fruits n'est pratiquement pas atteinte, les Etats-Unis et l'Europe interdisent toute entrée d'un fruit affecté de cette maladie.
} 
tion vers l'Europe. Solari sera le premier en 1932. Il exportera à Anvers et Londres. Mais, étant donné que les fruits n'étaient pas transportés dans des chambres froides, seuls $40 \%$ arrivèrent en bonne condition. Les efforts répétés pour réaliser des exportations expérimentales vers la France, l'Allemagne et la Belgique ont été toujours accompagnés d'une amélioration continue de la qualité. La crise que traversa l'Europe avec la seconde guerre mondiale repoussa encore les tentatives d'exportation à l'année 1958.

Par contre, l'augmentation des espaces de production durant les années trente impliqua une augmentation des risques de diffusion des agents pathogènes sans que le pays ne fût vraiment préparé à affronter cette crise. Au début des années 40, apparut le virus nommé Tristeza qui réduisit sensiblement la production nationale d'agrumes. Les conséquences de ce virus furent drastiques sur deux points : réduction de la production empêchant la surproduction et donc la possibilité de poursuivre la course aux marchés européens ; mais la conséquence la plus importante fût la réaction de l'Argentine qui, face à la propagation $\mathrm{du}$ virus, ferma ses portes aux agrumes uruguayens.

A nouveau, l'espace du Rio Uruguay qui avait joué un rôle moteur dans la production d'oranges, leur circulation et leur commercialisation se retrouva cantonné à son rôle de frontière en ce qui concerne ces fruits. Ce contexte peu stimulant impliqua de fait un repli des producteurs sur eux-mêmes et une période plutôt sombre en termes d'innovation et d'échanges technologiques encourageant davantage une conception individualiste parmi les producteurs. C'est dans ce contexte que Salto perdit l'hégémonie de la production de l'orange. A cette époque de prospérité de Salto, va succéder une période de croissance industrielle de la région de Paysandú, localisée un peu plus au sud. Cette nouvelle région va se développer en prônant un nouveau schéma de production. Ce développement, comme nous allons le voir, sera porté par un autre réseau ethnique.

\section{Le nouvel élan de l'exportation : l'émergence des pieds-noirs de Paysandu, la consolidation d'un complexe uruguayen d'agrumiculture.}

Pendant la période précédente, l'Etat avait été relativement absent de la promotion et du développement de la production agrumicole, mis à part une loi de Défense Agricole en 1911, dont la fonction essentielle était de délimiter et de réprimer l'invasion ou propagation d'animaux ou de végétaux préjudiciables 9 .

Dans la ville de Paysandú, un petit district industriel, plus particulièrement agro-industriel, s'était développé grâce à l'intervention de l'Etat. C'est en relation avec cette zone que se développe la production d'oranges. Ce développement est le fait d'immigrants français en partance d'Afrique du Nord suite à la décolonisation et qui viennent s'installer en Uruguay. La plupart d'entre eux optèrent pour l'Uruguay. Il choisirent ce pays après avoir été au Brésil et en Argentine pour sa stabilité politique. De plus, le pays était en plein développement et toutes les conditions étaient réunies pour développer la production d'oranges. Dans leur majorité, ces migrants avaient été producteurs de vigne et de

\footnotetext{
${ }^{9}$ En 1912, pendant le mandat de Battle y Ordoñez, une station agronomique est installée à Salto. Les écoles d'agronomie sont réorganisées et transformées en faculté d'agronomie de l'Université de la Républica en 1921. En 1925, est créée une Direction d'agronomie au Ministère de l'Industrie. En 1928, enfin, des laboratoires orientés vers l'expérimentation agricole voient le jour à Salto (voir l'article de Tanguy et Argenti dans ce numéro).
} 
fruits, ils détenaient un capital qui leur permit d'acheter des terres et de faire des investissements importants, tant dans la plantation que dans l'installation d'infrastructures.

L'option prise en faveur plutôt des oranges que de la vigne était liée dès le départ à un projet d'exportation vers l'Europe. C'est ainsi que cette colonie de français d'Algérie, et quelques autres européens présents sur la zone, plantèrent environ 2800 hectares d'orangers dans la région de Paysandú en 1956. Ce sont ces plantations qui permirent de fournir l'essentiel des exportations qui partirent vers l'Europe en 1962. Les exportations avaient effectivement repris dès 1958 avec Solari, mais c'est la colonie franco-algérienne qui fut l'acteur principal de l'exportation uruguayenne.

A nouveau, cette vague d'exportation a été confrontée à des problèmes d'organisation de la circulation de la marchandise, à des exigences en termes de réglementation et de contrôle financiers et fiscaux, et tout simplement à des problèmes d'adaptation aux usages et coutumes de la négociation du fruit dans le monde. A nouveau, bien sûr, l'hégémonie de la production de la viande et de la laine fixait les modèles, la réglementation et la dynamique des services et contrôles de l'Etat. La production de fruits exigeait une autre logique en termes de réglementations et d'exportations qui obligea les producteurs à mener des actions corporatives pour transformer ces règles.

Il fallait une révision globale du système agro-industriel agrumicole pour pouvoir mener à bien les exportations. Les revendications de cette communauté pied-noir s'organisèrent en dénonçant toute une série de problèmes que l'Etat devait selon eux finir par prendre en compte. La spécificité des problèmes de l'agrumiculture mettait en évidence, une fois de plus, la nécessité de penser cette culture de façon différente des normes instituées pour l'élevage. Les problèmes rencontrés par les producteurs étaient de divers types. Tout d'abord, ils devaient faire face à des problèmes techniques et logistiques concernant les emballages, il était nécessaire d'intégrer dans le process de l'activité de packing, les calibrages des produits et les bains de fongicide.... Une fois les produits emballés, une des questions les plus cruciales était celle de la conservation et des espaces réfrigérés. Ici, les éléments les plus problématiques ne concernaient pas tant les techniques et leur acquisitions que les acteurs avec lesquels il fallait travailler, par exemple les fournisseurs de fongicides, d'étiquetage, d'emballage, et plus encore les acteurs des flux maritimes qui ne maitrisaient pas les produits qu'ils transportaient et ne s'adaptaient pas aux petits volumes de produits exportés par l'Uruguay.

A cette époque, les confrontations avec les dockers, les armateurs, les arrimeurs et autres corporations de travailleurs ou bien encore avec les douaniers étaient continuelles. Pour les producteurs d'oranges, ces acteurs ne prenaient nullement en compte le fait que le produit avait une conservation limitée. Mais les producteurs s'opposaient également à l'inspection sanitaire et à la bureaucratie de l'Etat qui niaient totalement les temporalités courtes exigées dans la production agrumicole.

Ce grand nombre de problèmes impliqua une complexification croissante du système de production, de flux entre la production et la consommation et finalement de la commercialisation. Le fait de voir apparaître de plus en plus d'acteurs, dont certains étaient complètement extérieurs au monde de l'agrumiculture, provoqua des tensions notamment lorsqu'il s'agît de définir les compétences et les espaces d'intervention. C'est dans ce cadre qu'il fallut intégrer les importateurs/exportateurs parmi les acteurs clefs de la chaîne de production d'oranges, car ils possédaient les compétences nécessaires et inexistantes chez les producteurs d'oranges. 
A ce stade, l'exportation avait définitivement modifié la façon de produire et l'organisation du travail de l'agrumiculture. La complexité de l'activité d'exportation des fruits a marqué de façon très nette l'organisation en amont. Tout d'abord, les compromis et engagements de fourniture en début de récolte, la réservation des hangars et lieux de stockage sur les bateaux, tous ces obstacles eurent pour résultat d'aboutir à une juxtaposition des deux activités d'exportation et de production afin de les articuler au mieux des intérêts des clients.

Malgré cette difficulté, le système s'est peu à peu institutionnalisé atteignant une efficience raisonnable, notamment avec la création en 1970 de la Commission Honoraire Nationale du Plan Citricole (ou agrumicole) (CHNCP). Sans rentrer dans le détail de ce Plan, on peut dire qu'il répondait en grande partie à toutes les revendications portées principalement par la communauté Pied-Noir de Paysandú.

\section{Conclusion :}

Dans cet article nous avons reconstruit l'histoire de l'orange en présentant de façon articulée les étapes de développement qui nous ont permis de montrer que, dès son arrivée, l'orange a été le «fruit » d'une «mondialisation». Il s'agissait tout d'abord d'une division internationale du travail privilégiant l'hégémonie de certains marchés. Les dispositifs de l'époque mettaient en œuvre les prémices d'une réglementation organisant déjà le marché et ses contraintes avec une forte prégnance des rapports Nord/Sud.

Nous avons également mis en exergue le fait que ces marchés se sont construits dès le départ en s'appuyant sur des réseaux sociaux avec le rôle majeur joué par les jésuites, les italiens, les pieds-noirs : la circulation des hommes entre deux mondes a permis la circulation des savoirs-faire et des outillages nécessaires à la production.

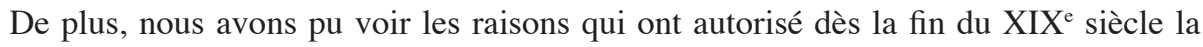
constitution de formes ou modèles d'entreprises déjà complexes, allant des simples producteurs aux propriétaires de la chaîne de production dans son ensemble.

Enfin, nous avons pu situer cette histoire dans le cadre de celle des transports, qui contribuent fortement à orienter les modes de production et la localisation de la production, privilégiant les périphéries des deux pays le long du fleuve Rio Uruguay et «sacrifiant $\gg$ les producteurs du cœur du pays.

Terminons en insistant sur le fait que deux logiques complémentaires apparaissent :

La première, celle de la recherche continue de la part des migrants de l'établissement de règles stables internes aux entreprises et des différents échelons de la chaîne de production/consommation. Cette logique est le produit de liens sociaux mettant en oeuvre des systèmes relativement solides entre les différents acteurs (réseau ethnique) qui partagent petit à petit ce système de production/consommation au cours du temps. Nous l'avons présenté via une reconstitution chronologique des acteurs de l'orange. Précisons que n'étant pas des historiens nous avons mobilisé ce qui nous permettait d'expliquer au cours de cette chronologie les évolutions dans la façon de produire l'orange et la construction de l'espace organisationnel qui est en lien avec cette production.

La seconde logique est celle qui est significative d'une ouverture constante vers la nouveauté et l'efficacité avec le but sans cesse recherché d'élaborer le meilleur produit possible. Nous avons mis l'accent sur cette capacité constante de la part des différentes communautés de transformer, en s'appropriant des améliorations techniques, en réajus- 
tant sans cesse les relations sociales porteuses des avancées techniques. En développant une capacité permanente d'apprentissage et en innovant techniquement, organisationnellement et socialement, les producteurs d'orange ont toujours essayé de se maintenir à des niveaux de qualité exigés par le marché.

\section{Références}

Barbato Celia (Coordinatrice), 2000, Mercosur, una estrategia de desarollo, Nuevas miradas desde la economia y la politica, Ediciones Trilce, 85 pages.

Dentesano Pericles, Regali Enzo Alberto, 2000, El Mercosur y la region centro - la hidrovia parana-paraguay - el corredor austral, Ediciones del Corredor Austral, 123 pages.

Neffa Julio Cesar, 2000, Que hacer para mejorar la competitividad de las PYME Argentinas?, Asociacion Trabajo y Sociedad, Programa de Investigaciones Economicas sobre Tecnologia, Trabajo y Empleo (CEIL - PIETTE CONICET), 245 pages.

Mercier D. et Tanguy C. «Entre homogénéisation par les normes et logiques d'action différenciées : la production d'Oranges en Argentine et en Uruguay», $\mathrm{N}^{\circ} 25$, série socio-économie du travail, Revue Economies et Sociétés.

Santi I. « Migrations en Argentine II » in Les cahiers ALHIM, N9, 2004.

Censo Nacional Citricola 1996, Comision Honoraria Nacional del Plan Citricola, 1997. 
